\title{
Multiscale Molecular Modeling in G Protein-Coupled Receptor (GPCR)-Ligand Studies
}

\author{
Pratanphorn Nakliang, Raudah Lazim, Hyerim Chang and Sun Choi *(1) \\ College of Pharmacy and Graduate School of Pharmaceutical Sciences, Ewha Womans University, \\ Seoul 03760, Korea; pnakliang@ewha.ac.kr (P.N.); raudah@ewha.ac.kr (R.L.); hrchang@ewhain.net (H.C.) \\ * Correspondence: sunchoi@ewha.ac.kr; Tel.: +82-2-3277-4503; Fax: +82-2-3277-2851
}

Received: 5 March 2020; Accepted: 17 April 2020; Published: 19 April 2020

\begin{abstract}
G protein-coupled receptors (GPCRs) are major drug targets due to their ability to facilitate signal transduction across cell membranes, a process that is vital for many physiological functions to occur. The development of computational technology provides modern tools that permit accurate studies of the structures and properties of large chemical systems, such as enzymes and GPCRs, at the molecular level. The advent of multiscale molecular modeling permits the implementation of multiple levels of theories on a system of interest, for instance, assigning chemically relevant regions to high quantum mechanics (QM) level of theory while treating the rest of the system using classical force field (molecular mechanics (MM) potential). Multiscale QM/MM molecular modeling have far-reaching applications in the rational design of GPCR drugs/ligands by affording precise ligand binding configurations through the consideration of conformational plasticity. This enables the identification of key binding site residues that could be targeted to manipulate GPCR function. This review will focus on recent applications of multiscale QM/MM molecular simulations in GPCR studies that could boost the efficiency of future structure-based drug design (SBDD) strategies.
\end{abstract}

Keywords: G protein-coupled receptors (GPCRs); multiscale calculations; molecular modeling; structure-based drug design (SBDD)

\section{Introduction}

G protein-coupled receptors (GPCRs) have been known as the largest family of human membrane protein that plays crucial roles in many biological processes such as vision, sensing, and neurotransmission [1-8]. Signal transmission through GPCRs is initiated by the binding of extracellular ligands including drugs, hormones, and other stimuli. The structural dynamics of GPCR as a consequence of ligand binding is considerably complex and contribute to its physiological functions. At the orthosteric binding site, GPCR ligands can be roughly divided into agonists and antagonists in which the first one activates the GPCR activity, whereas the latter act as blockers. Besides this, GPCR ligands can also bind to the allosteric site and indirectly affect the agonistic activity of GPCRs. Therefore, examining for ligand-receptor interactions that are vital in effectuating desirable GPCR functions has been the mainstream focus of most GPCR studies. To date, GPCRs are a target of more than $30 \%$ of approved drugs $[9,10]$. The understanding of the effects of ligands on GPCR properties has become the main task in rational drug and ligand designs. The importance of GPCR as viable drug targets was portrayed by the conferment of the Nobel Prize in Chemistry to Lefkowitz and Kobilka in 2012 for their groundbreaking discoveries on GPCRs [11]. The increase in structural data and the exploration of GPCR dynamics revealed the flexibility of its binding pockets as well as the tendency of GPCRs to adopt distinct conformations at different states. Therefore, computational simulation could serve as a complementary tool that could help in the discovery and design of GPCR ligands with desirable effects by permitting the scrutiny of GPCR dynamics. 
Acquiring a comprehensive understanding of biomolecular drug interactions is essential in drug discovery. Many biological and biophysical techniques, such as X-ray crystallography, electron microscopy, cryo-electron microscopy (cryo-EM), small-angle X-ray scattering (SAXS), neutron scattering (SANS), and nuclear magnetic resonance spectroscopy (NMR), have been utilized to solve the three-dimensional conformations of proteins [12]. Currently, a large number of biological information has been published, providing resources required to probe proteins in silico, at the atomic level [5,6,13-15].

Molecular simulations provide the atomistic-level details useful for revealing and predicting important determinants influencing drug design such as binding affinity, reaction mechanism, and protein-ligand interactions. Molecular dynamics (MD) simulation, in particular, is becoming a powerful computational approach that applies classical molecular mechanics (MM) potential to simulate the time-dependent motions of atoms in the system of choice, hence providing researchers with the means to sample myriads of conformations that a protein may assume during biological processes at reasonable computational complexity. The MM method considers atoms and bonds as balls and springs, respectively. The physical properties and energy function of a system are calculated using force fields that are described by Newtonian mechanics. The cost-effectiveness of MD simulation saw its application to a wide variety of protein studies related to protein folding and unfolding, protein stability, structural transition, protein adaptation to different environments, and protein-ligand binding kinetics. However, an apparent setback of classical MM force field is its mean-field treatment of amino acid residues, hence rendering MD simulation unsuited for the elucidation of chemical reactions, which requires an accurate representation of the electronic structure of the system. This can be achieved via solving the Schrödinger equation in quantum mechanics (QM) calculation, a highly accurate computational approach that can be used for structure optimization, mechanistic studies of chemical reactions, and modeling of the optical properties of light-sensitive proteins, among many other uses. Contrary to MM force field, a limitation of the QM method is high computational demand and, therefore, is not appropriate for large systems ( $>$ hundreds of atoms) [12].

Structure-based drug design (SBDD) is gaining tremendous attention for its potential in contributing to the design of novel drug candidates. With the continuous solving of the 3D conformations of GPCRs, a thorough understanding of the structural features of the receptors, such as orthosteric and allosteric binding site configurations, structural flexibility, and domain motions, can be attained and utilized in SBDD strategies for the development of novel GPCR drugs with ameliorated efficacy, efficiency, and specificity. The application of MD simulations in GPCR studies has been broadly reported, giving useful insights into ligand-binding interactions and GPCR dynamics [4-7]. However, to obtain a better picture of the binding site conformation as well as the chemical properties of ligands bound to GPCRs, more accurate descriptions of binding sites are necessary. Multiscale simulations are combinations of different time and length-scale resolution techniques (Figure 1). These techniques can be used at the same time or as a complementary method. The application of multiscale QM/MM simulation will be highly desirable for this purpose since this hybrid approach combines the advantages of both classical and QM theory, enabling the modeling of large complex chemical systems at a higher level of sophistication without exhausting too much computational resources [16-18]. In QM/MM molecular modeling, the enormous biological molecule will be divided into two (or more) regions, whereby a small region of interest (e.g., binding site) is described by the accurate QM method while the rest of system (protein, membrane, solvent, etc.) is treated using the less expensive MM level (Figure 2). Some examples of QM/MM applications in drug discovery include inhibitor design from transition state and/or intermediate structures, mechanism-based inhibitor design, prediction of drug metabolism, and observation of changes in the chemical nature of ligands due to protein conformational transitions. In 2013, the Nobel Prize in Chemistry was awarded to Karplus, Levitt, and Warshel for their contributions in developing multiscale molecular modeling for complex chemical systems, including biological molecules [19]. 


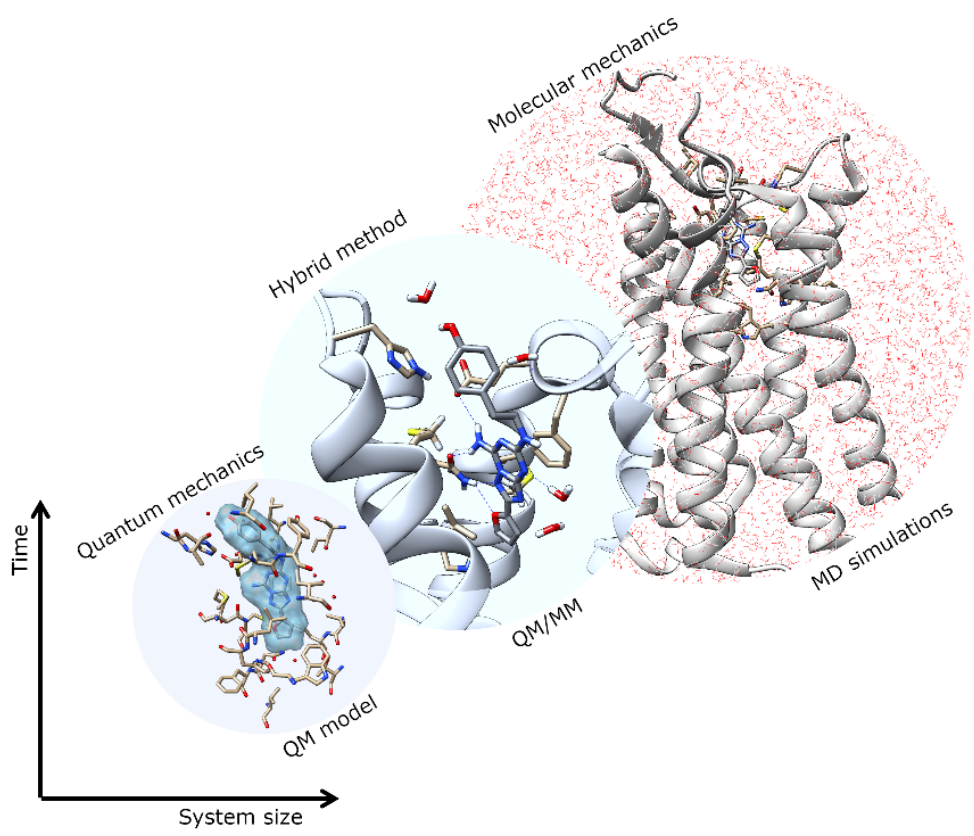

Figure 1. Multiscale modeling and application in computational simulations.

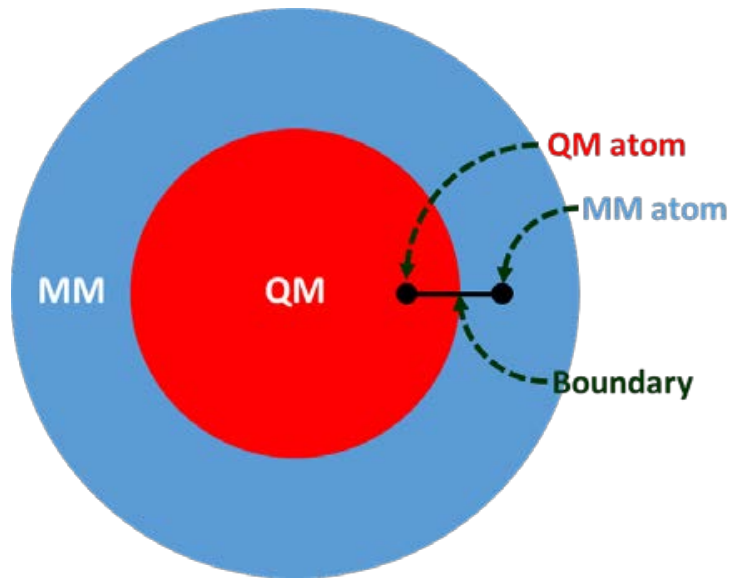

Figure 2. The hybrid quantum mechanics/ molecular mechanics (QM/MM) system partition and atoms at the QM-MM boundary.

\section{Molecular Docking Development using QM/MM Approach}

The docking approach is a promising tool that is commonly utilized in SBDD efforts as a means to predict binding conformation of small molecules in the binding sites of target proteins. A number of docking software is available, for example, AutoDock [20], GOLD [21], GLIDE [22-24], and SwissDock [25], thus enabling facile determination of potential binding poses of ligands in proteins. The principle of docking simulations is based on the "lock-and-key" hypothesis, wherein the protein (host) is the lock while the ligand (guest) is the key, hence implying the specificity of a ligand to bind to a certain protein. During docking simulations, the orientation of guest(s) in the host is optimized and the accuracy of this process depends on two major components namely searching algorithm and scoring function [26]. Additionally, consideration of protein and ligand flexibility has been shown to play a crucial role in improving the accuracy of predicting ligand binding affinity to target protein. Thus, the efficiency of the docking method will be improved by including protein flexibility using induced-fit docking (IFD) or utilizing more than one host structure by acquiring a protein ensemble through MD simulations [27-30]. Recently, the QM/MM method was employed to improve the quality of docking simulations. The advantage of this approach is that the flexible protein environment is 
considered during the QM/MM calculation through simple geometry optimization of the assigned QM region in the presence of the free energy surface of the surrounding protein, which was constructed based on MM potential. The accuracy of the protocol was validated using a set of known protein-ligand structure complexes, which was taken from experimental data.

The implementation of QM/MM into docking protocol has been developed by many research groups to improve the accuracy for use in structure refinement and routine analysis involved in docking studies. The binding conformation of the ligand within the binding pocket of olfactory receptor MOR244-3 was studied [31]. The ligand-binding site contains a $\mathrm{Cu}(\mathrm{I})$ ion, which is responsible for the binding of the organosulfur odorant. In the study, the ligand-protein and ligand-Cu interactions are well characterized by the QM/MM description in which the QM region covers all important residues in the binding pocket. The calculated results are consistent with the mutagenesis studies of the receptor activation, which showed that the binding site consists of the $\mathrm{Cu}$ ion coordinating with His105, Cys109, and Asn202. Additional analyses performed using various ligands revealed that the thioether group is a significant part of the ligand-binding mechanism. The obtained results could be applied as a case study for other mammalian olfaction investigation. Recently, the activation of human odorant receptors, OR5AN1 and OR1A1, was studied to compare the calculated binding energies of (R)-muscone and other related compounds [32]. The theoretical results are in good agreement with the experimental results that indicate the preference for $(R)$ - over $(S)$ - enantiomer. Structural observation revealed that the ligand is stabilized by forming a hydrogen bond with Tyr260 and hydrophobic interactions with surrounding aromatic residues. This valuable finding may lead to the instructive development of the quantitative structure-activity relationship (QSAR) model. QM/MM simulation has also been utilized to improve the quality of the docking results of human dopamine D3 receptor (D3R), which has been identified as an antipsychotic drug target for schizophrenia treatment [33,34]. The well-known atypical antipsychotic (AAP) drugs include risperidone, aripiprazole, ziprasidone, clozapine, olanzapine, and quetiapine. All of these have been prescribed to treat various mental conditions [35]. The QM/MM minimization was performed on the selected docking poses. Only the ligand (haloperidol) was placed in the QM region, while the rest of the system was considered as the MM region. Accuracy of the interaction energy was shown to be dependent on the radius of the binding site that was included in the QM region during the calculation. It was due to the long-range interactions of distant charged residues that were included in the QM region. The interaction energy was calculated as $-170.1 \mathrm{kcal} / \mathrm{mol}$, which was larger than the other two classical methods used $(-56.3 \mathrm{kcal} / \mathrm{mol}$ for classical mechanics minimization of all hydrogen atoms and haloperidol molecule, and $-137.6 \mathrm{kcal} / \mathrm{mol}$ for only hydrogen atoms minimization). It indicated that the QM/MM refinement converged to the more stable conformation than classical minimization techniques. The combination of docking and QM/MM calculation revealed the important roles of surrounding amino acid residues in the binding pocket. Moreover, the hydroxyl group of haloperidol was identified as a major site that leads to stronger binding to dopamine receptors.

In 2005, the QM/MM simulation was incorporated into the docking algorithm, whereby the fixed charges of ligand assigned by MM force fields were replaced by partial charges fitted based on electrostatic potential of the ligand derived in the presence of protein environment during QM/MM calculation. Here, the ligand was the only molecule assigned as the QM region, while the rest of the system were described using the MM potential [36]. Cho et al. found that the use of polarized charges plays a significant role in improving the prediction of ligand binding mode and this leads to a new promising docking protocol for lead optimization in drug discovery. A subsequent study on metalloproteins suggested that the extension of the QM region to include metal ion(s) along with coordinated protein residues is important and leads to more reliable binding poses [37]. Due to the success of the incorporation of QM/MM into docking simulation, its applications in structure-based studies, including drug design, virtual screening, and lead optimization, have been investigated [38-40]. Current assessment of GPCR docking simulations without QM/MM showed that the success rate was over 70\%. Docking error was evident especially for the docking of ZM241385 and XAC into Adenosine 
$\mathrm{A}_{2 \mathrm{~A}}$ receptor [41,42]. In 2016, Kim and Cho incorporated QM and solvation effect into the docking simulation of GPCRs to improve the predicting accuracy. They proposed a new docking protocol that replaced the fixed force field charges of the ligand by partial charges calculated using QM/MM calculations with an extended QM region. This protocol was also used in re-docking simulations. The QM region used in the study included the ligand and surrounding amino acid residues within $5 \AA$ of the ligand. The solvation effect was taken into account by solving the Poisson Boltzmann (PB) equation. Among a test set of $40 \mathrm{GPCR}$-ligand complexes, the QM/MM docking improved the success rate to $90 \%$ without solvation effect, which is better than the docking result from Glide with standard precision (Glide SP) and Glide with solvation effect included. The improvements in docking poses are shown in Figure 3. A possible issue of failed cases is the ligand containing solvent-exposed part(s). Therefore, integration of solvent effect into the $\mathrm{QM} / \mathrm{MM}$ docking protocol by using an implicit solvent model was proposed. It demonstrated an excellent improvement with a success rate of $100 \%$, portraying the importance of charge models in improving docking accuracy [43].

A.

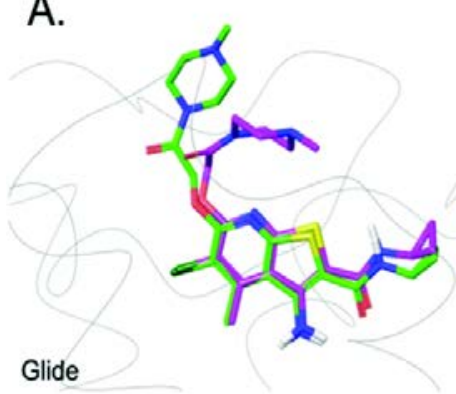

C.

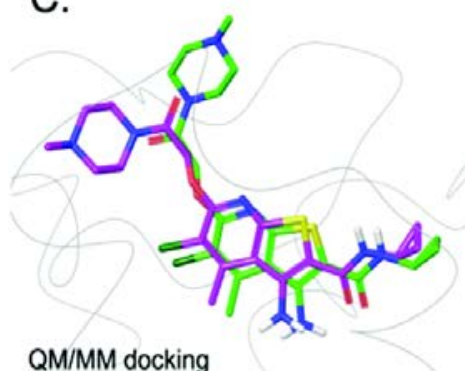

B.

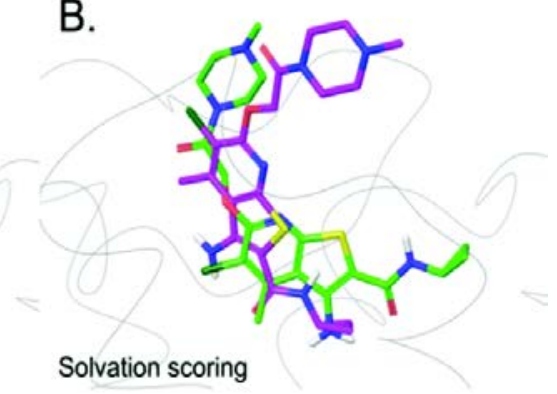

D.

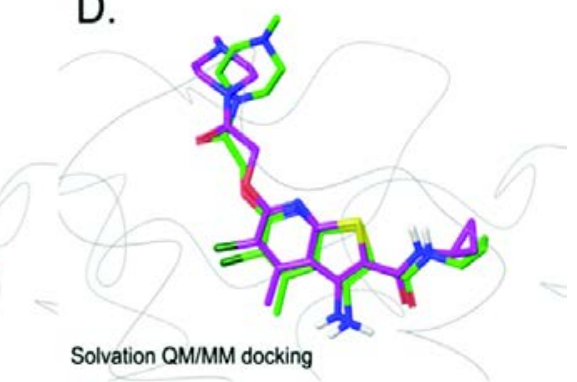

Figure 3. Prediction of LY2119620 binding modes using the four docking methods (green - crystal pose, purple - predicted pose). (A) Glide docking result. (B) Solvation scoring result. (C) QM/MM docking result. (D) Solvation QM/MM docking result. Heteroatoms are shown in yellow $(\mathrm{S})$, red $(\mathrm{O})$, blue $(\mathrm{N})$, and dark green $(\mathrm{Cl})$. Reproduced from Reference 43 with permission from the PCCP Owner Societies.

\section{Class A Rhodopsin Photoactivity Investigation}

Class A rhodopsin receptor is responsible for many physiological functions, particularly light-sensitive responses [6]. The biological activity of rhodopsin is initiated by light (photon energy). As a result of photoisomerization, it is possible to use rhodopsin as an energy storage material [44]. Therefore, photochemical events are the main topic of interest in most studies related to this receptor. The complete picture of photochemical reactions could be achieved computationally. A suitable computational method for rhodopsin photoactivity investigations is the QM/MM method that has been applied to understand structure, spectral tuning, photoisomerization, and mutations. Photoexcitation calculations demand high computational resources. Thus, retinal chromophore that is covalently bound to activated rhodopsin has been studied using many small models of the ligand to understand the rapid photoisomerization process $[45,46]$. Even though the gas phase calculated excitation energies are in good agreement with experiment, the effect of protein on the photochemical reaction was not explained using these models, especially the steric effects on the -ionone ring. 
Methods aimed at understanding the effect of the protein environment on the photochemical process occurring during rhodopsin activation have been developed. The availability of high-resolution structural data accelerates the theoretical studies involving the structure-function relationship of rhodopsin. The photoisomerization of 11-cis rhodopsin to all-trans bathorhodopsin is one of the most attractive properties that has been widely investigated. A variety of hybrid methods have been used ranging from simple to complicated QM calculations. The energy difference between the minimum energies of rhodopsin and bathorhodopsin yielded energy storage of $34.1 \mathrm{kcal} / \mathrm{mol}$, as calculated using QM/MM method at the B3LYP/6-31G*:AMBER level of theory. The result is in excellent agreement with experimental data [47-49]. The energy decomposition analysis revealed that large energy storage is due to the electronic interaction of rhodopsin. The rotation of the C11-C12 dihedral angle from $-11^{\circ}$ in 11 -cis rhodopsin to $-161^{\circ}$ in all-trans bathorhodopsin was driven by the steric interaction between Ala117 and the polyene chain at the $\mathrm{C} 13$ position. This steric interaction hindered the rotation of the C11-C12 dihedral angle toward positive angles, an occurrence which could not be observed in the gas phase model. This study indicated that Glu113 may act as a counterion. Moreover, they suggested that the salt bridge between NH of the Schiff base linkage and Glu113 may be an important factor that influenced the electrostatic contribution of the protein to the total energy storage. The polarized bond at the Schiff linkage of bathorhodopsin shifted away from the negative site of Glu113 as compared to rhodopsin. The electrostatic contribution analysis of nearby residues in the binding pocket also provided insights on individual interactions, revealing that Ala117, Ser186, and a water molecule may stabilize bathorhodopsin relative to rhodopsin. The electronic-excitation energy estimation was also improved due to the integration of the electrostatic contribution of the protein environment during energy calculations.

With the increment in the number of available experimental GPCR structures, subsequent theoretical studies on rhodopsin have been in the spotlight [50-53]. In 2010, the structure and properties of squid rhodopsin were investigated. Similar to bovine rhodopsin, it contains 11-cis rhodopsin covalently bonded to Lys305. However, Glu113 in bovine rhodopsin is replaced by a group of Asn87, Tyr111, Glu180, and a water molecule. At that time, the position of internal water molecules could not be determined by X-ray crystallographic studies. Therefore, the number and positions of internal water molecules were verified by QM/MM calculation. It was found that the calculated structure of two additional water molecules near the Schiff base region is in good agreement with the X-ray structure. The absorption wavelength of retinal-chromophore blue-shifted around $120 \mathrm{~nm}$ when protein polarizability was accounted during the calculation. The effect of particular residues within $4 \AA$ of the retinal polyene chain (34 amino residues) toward photoactivity of 11-cis rhodopsin was calculated by turning off the charges of these residues, one at a time. Among these residues, Glu180 blue-shifted the absorption wavelength by around $100 \mathrm{~nm}$ and was identified as the main counterion in squid rhodopsin. They suggested that even though Glu180 is located further away from the retinal chromophore compared to Glu113 in bovine rhodopsin, the charge stabilization engendered by Glu180 still has a significant effect on the optical properties of squid rhodopsin.

The QM/MM calculation of Class A rhodopsin GPCRs also provided a new perspective on retinitis pigmentosa, a disease involving progressive retinal degradation [54,55]. Rhodopsin mutations have been identified as a major cause of this disease [56]. Therefore, many mutagenesis studies have been conducted to determine key residues that may contribute to the development of retinitis pigmentosa. However, the mechanisms and causes of mutation are not clear. Hernández-Rodríguez et al. studied two mutated human rhodopsins (S186W and M207R) and compared the mutated models to that of wild type. The protein models were solvated in water and phosphatidylcholine (POPC) lipid bilayer. A combination of various computational methods, namely MD simulations, density functional theory (DFT), and QM/MM, was applied. The results unveiled that a less stable counterion region could impair the whole protein in the mutated models. Moreover, the strong blue-shift resulting from the mutations leads to excess energy that could yield side reactions. The results of this study could be utilized to support the rational development of medical treatment. 
Besides the effect of protein environment, the structure of the retinal ligand itself also plays an important role in photoisomerization. The cis-trans isomerization of rhodopsin and isorhodopsin was studied using a combination of QM/MM and MD simulations [57]. Isorhodopsin is a rhodopsin analog that has a 9-cis retinal chromophore instead of an 11-cis retinal chromophore. MD simulations suggested that isomerization is a fast and facile event in rhodopsin, while being a much more complicated phenomenon in isorhodopsin. The 9-cis position in the retinal ligand of isorhodopsin forms a steric hindrance within the narrow space inside the opsin, thus affording byproducts. QM/MM calculations simulating the photoactivity of both systems showed that isorhodopsin photoisomerization gave rise to alternative products such as the 9,11-di-cis isomer. This is in contrary to the straightforward bathorhodopsin-only pathway in rhodopsin isomerization. Therefore, rhodopsin is preferred in nature. According to the simulations, protein environments, counterion, and chromophore structures are key factors that governed the photoactivity of rhodopsin. Incorporation of QM/MM simulations would broaden the understanding of particular state of rhodopsin photoactivation related diseases. The obtained knowledge can be utilized in drug design that target to stabilize the degradation of rhodopsin [58].

Currently, many complicated simulations are accessible. As mentioned above, the function of rhodopsin depends on many factors and understanding the protein-ligand interactions of rhodopsin is vital for the rational design of novel ligands and biomimicking molecules. The automatic rhodopsin modeling (ARM) method was proposed to study and predict the optical properties of class A rhodopsin system [59]. The protocol of this theoretical tool is as follows; (i) chromophore cavity definition, (ii) protonation state of amino acid residues, (iii) counterion position, and (iv) appropriate generation of mutation residue(s) for further parallel studies. Based on their benchmark test set, the computational maximum absorption wavelength $\left(\lambda_{\text {max }}^{a}\right)$ showed excellent agreement with observed experimental data. As a result, automatic a-ARM provides high reproducibility (user-independent). Moreover, the utilization of ARM reduced the preparation time and also provided a practical simulation protocol for rhodopsin and other classes of GPCRs. The detailed structure-function and energetic analysis will provide a complete picture of the class A rhodopsin and also mutation-specific therapies.

\section{The QM Approach in GPCR Studies}

Recently, an approximate molecular orbital (MO) method called Fragment Molecular Orbital (FMO) was implemented into studies related to GPCR-ligand interactions [60-68]. FMO has been described in previous publications and review articles [60-62]. Therefore, only a brief introduction of this method is presented here. The modus operandi of FMO involves the division of the system into fragments followed by QM calculations of each fragment. This method reduces the amount of time required to conduct QM calculations of the whole system. The interaction between two fragments is characterized by electrostatics, exchange-repulsion, charge transfer, and dispersion interactions (Figure 4). Therefore, the application of FMO on GPCR-ligand studies would yield reliable protein-ligand interactions that are important for biomolecular recognition. The information obtained is useful for SBDD. Weak interactions such as halogen bonds, cation-interactions, and non-classical hydrogen bonds that could not be explained by the MM force field could be achieved through the FMO method. These interactions have been shown to be key features in biological processes such as ligand recognition and protein folding. The theoretical characterization of ligand-binding recognition in GPCRs exhibited similar electrostatic and hydrophobic interactions across most GPCR complexes. In 2016, Heifetz and coworkers performed the FMO calculation on the complexes of agonist-orexin-2 receptor $\left(\mathrm{OX}_{2} \mathrm{R}\right)[64]$. They considered all interactions with an absolute pair interaction energy (PIE) greater than or equal to $3.0 \mathrm{kcal} / \mathrm{mol}$. A comparison of the interactions of two docking poses indicated that they shared similar interactions, and this was supported by site-directed mutagenesis studies. Subsequently, GPCR-ligand crystal structures were investigated [65]. They revealed the often omitted interactions contributed by surrounding residues, especially hydrophobic interaction and the involvement of backbone atoms. Comprehensive QM studies of protein-ligand interactions provide valuable information for rational 
SBDD. For instance, which ligand fragments could be targeted for modification to achieve desired properties [68]. Data on protein-ligand interactions acquired based on the FMO method have been published online (https://drugdesign.riken.jp/FMODB/) [69]. Currently, more than 980 unique PDB entries were identified. Moreover, an automated FMO calculation protocol was also developed in 2019 [70]. It is a valuable guideline for mutagenesis, interaction studies, and protein engineering.

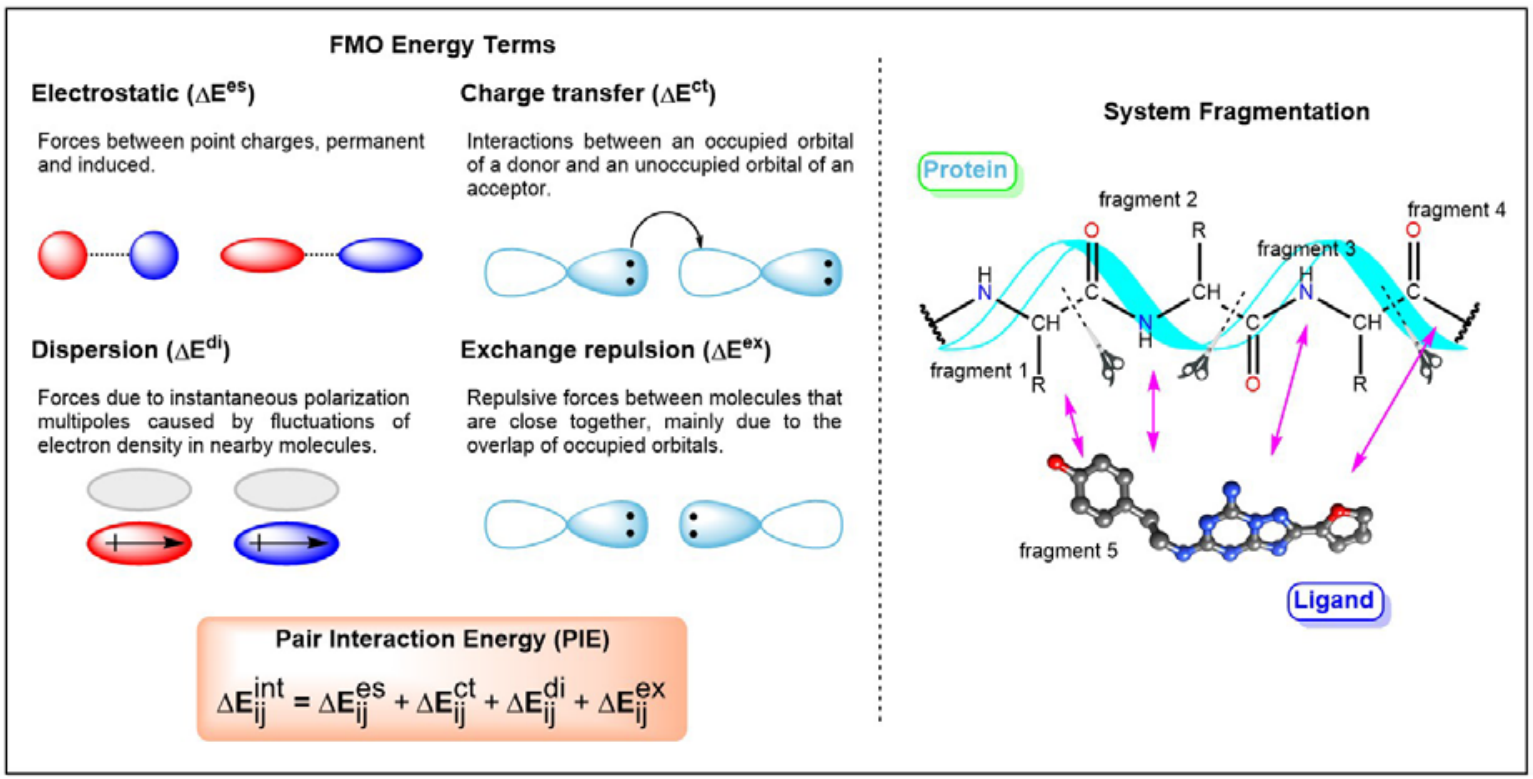

Figure 4. Workflow of the fragment molecular orbital (FMO) method and details of pair interaction energy (PIE) terms. Adapted from Reference 64 with permission.

\section{Conclusions and Outlooks}

GPCRs are important membrane proteins that play key roles in numerous physiological processes. GPCR-ligand (drug) interactions are crucial in modulating GPCR activity. Thus, a detailed understanding of GPCR-ligand interaction is needed for the design and development of new GPCR therapeutics. Previously, most of SBDD efforts in GPCR studies employed classical molecular docking that allows researchers to achieve their goals effectively. However, the development of multiscale molecular modeling has resulted in the reduction of computational demand for a relatively high-level accuracy approach such as QM. The application of a sophisticated computational strategy to a large and complex GPCR system was made feasible through QM/MM method, thus providing a practical prediction method that offers new insights into the structure, interaction, dynamics, and kinetics of GPCRs. Furthermore, the incorporation of QM in calculations provides missing pieces of important weak protein-ligand interactions such as hydrogen bond, cation- $\pi$, and non-classical hydrogen bond, which could not be determined by classical MM methods. Thus, it will improve the current SBDD protocol, making it valuable for pharmaceutical research in the near future.

Author Contributions: P.N., R.L., H.C., and S.C. conceived and wrote the papers; P.N., R.L., and H.C. gathered literature and organized the figures and tables; all authors contributed to the editing of the manuscript. All authors have read and agreed to the published version of the manuscript.

Funding: This work was supported by Bio and Medical Technology Development Program (NRF-2019M3E5D4065251) funded by the Ministry of Science and ICT (MSIT) and the Ministry of Health and Welfare (MOHW) through the National Research Foundation of Korea (NRF), Medical Research Center (MRC) grant (No. 2018R1A5A2025286), and the Mid-career Researcher Program (NRF-2017R1A2B4010084).

Conflicts of Interest: The authors declare no conflict of interest. 


\section{References}

1. Basith, S.; Cui, M.H.; Macalino, S.J.Y.; Park, J.; Clavio, N.A.B.; Kang, S.; Choi, S. Exploring G Protein-Coupled Receptors (GPCRs) Ligand Space via Cheminformatics Approaches: Impact on Rational Drug Design. Front. Pharmacol. 2018, 9, 1-26. [CrossRef] [PubMed]

2. Chan, H.C.S.; Li, Y.; Dahoun, T.; Vogel, H.; Yuan, S.G. New Binding Sites, New Opportunities for GPCR Drug Discovery. Trends Biochem. Sci. 2019, 44, 312-330. [CrossRef] [PubMed]

3. Heifetz, A.; James, T.; Morao, I.; Bodkin, M.J.; Biggin, P.C. Guiding lead optimization with GPCR structure modeling and molecular dynamics. Curr. Opin. Pharmacol. 2016, 30, 14-21. [CrossRef] [PubMed]

4. Latorraca, N.R.; Venkatakrishnan, A.J.; Dror, R.O. GPCR Dynamics: Structures in Motion. Chem. Rev. 2017, 117, 139-155. [CrossRef] [PubMed]

5. Yuan, X.J.; Xu, Y.C. Recent Trends and Applications of Molecular Modeling in GPCR-Ligand Recognition and Structure-Based Drug Design. Int. J. Mol. Sci. 2018, 19, 2105. [CrossRef]

6. Lee, Y.; Basith, S.; Choi, S. Recent Advances in Structure-Based Drug Design Targeting Class A G Protein-Coupled Receptors Utilizing Crystal Structures and Computational Simulations. J. Med. Chem. 2018, 61, 1-46. [CrossRef]

7. Lee, Y.; Lazim, R.; Macalino, S.J.Y.; Choi, S. Importance of protein dynamics in the structure-based drug discovery of class A G protein-coupled receptors (GPCRs). Curr. Opin. Struc. Biol. 2019, 55, 147-153. [CrossRef]

8. Chan, H.C.S.; Wang, J.J.; Palczewski, K.; Filipek, S.; Vogel, H.; Liu, Z.J.; Yuan, S.G. Exploring a new ligand binding site of $\mathrm{G}$ proteincoupled receptors. Chem. Sci. 2018, 9, 6480-6489. [CrossRef]

9. Santos, R.; Ursu, O.; Gaulton, A.; Bento, A.P.; Donadi, R.S.; Bologa, C.G.; Karlsson, A.; Al-Lazikani, B.; Hersey, A.; Oprea, T.I.; et al. A comprehensive map of molecular drug targets. Nat. Rev. Drug Discov. 2017, 16, 19-34. [CrossRef]

10. Sloop, K.W.; Emmerson, P.J.; Statnick, M.A.; Willard, F.S. The current state of GPCR-based drug discovery to treat metabolic disease. Brit. J. Pharmacol. 2018, 175, 4060-4071. [CrossRef]

11. The Nobel Prize in Chemistry 2012. Available online: https:/www.nobelprize.org/prizes/chemistry/2012/ summary/ (accessed on 10 February 2020).

12. Amaro, R.E.; Mulholland, A.J. Multiscale methods in drug design bridge chemical and biological complexity in the search for cures. Nat. Rev. Chem. 2018, 2. [CrossRef] [PubMed]

13. Hauser, A.S.; Chavali, S.; Masuho, I.; Jahn, L.J.; Martemyanov, K.A.; Gloriam, D.E.; Babu, M.M. Pharmacogenomics of GPCR Drug Targets. Cell 2018, 172, 41. [CrossRef] [PubMed]

14. Bondar, A.N.; Lemieux, M.J. Reactions at Biomembrane Interfaces. Chem. Rev. 2019, 119, 6162-6183. [CrossRef]

15. Burley, S.K.; Berman, H.M.; Bhikadiya, C.; Bi, C.; Chen, L.; Di Costanzo, L.; Christie, C.; Dalenberg, K.; Duarte, J.M.; Dutta, S.; et al. RCSB Protein Data Bank: Biological macromolecular structures enabling research and education in fundamental biology, biomedicine, biotechnology and energy. Nucleic Acids Res. 2018, 47, 464-474. [CrossRef] [PubMed]

16. Vreven, T.; Byun, K.S.; Komaromi, I.; Dapprich, S.; Montgomery, J.A.; Morokuma, K.; Frisch, M.J. Combining Quantum Mechanics Methods with Molecular Mechanics Methods in ONIOM. J. Chem. Theory Comput. 2006, 2, 815-826. [CrossRef] [PubMed]

17. Senn, H.M.; Thiel, W. QM/MM Methods for Biomolecular Systems. Angew. Chem. Int. Edit. 2009, 48, 1198-1229. [CrossRef] [PubMed]

18. Chung, L.W.; Sameera, W.M.C.; Ramozzi, R.; Page, A.J.; Hatanaka, M.; Petrova, G.P.; Harris, T.V.; Li, X.; Ke, Z.F.; Liu, F.Y.; et al. The ONIOM Method and Its Applications. Chem. Rev. 2015, 115, 5678-5796. [CrossRef]

19. The Nobel Prize in Chemistry 2013. Available online: https://www.nobelprize.org/prizes/chemistry/2013/ summary/ (accessed on 10 February 2020).

20. Trott, O.; Olson, A.J. Software News and Update AutoDock Vina: Improving the Speed and Accuracy of Docking with a New Scoring Function, Efficient Optimization, and Multithreading. J. Comput. Chem. 2010, 31, 455-461. [CrossRef]

21. Jones, G.; Willett, P.; Glen, R.C.; Leach, A.R.; Taylor, R. Development and validation of a genetic algorithm for flexible docking. J. Mol. Biol. 1997, 267, 727-748. [CrossRef] 
22. Friesner, R.A.; Banks, J.L.; Murphy, R.B.; Halgren, T.A.; Klicic, J.J.; Mainz, D.T.; Repasky, M.P.; Knoll, E.H.; Shelley, M.; Perry, J.K.; et al. Glide: A new approach for rapid, accurate docking and scoring. 1. Method and assessment of docking accuracy. J. Med. Chem. 2004, 47, 1739-1749. [CrossRef]

23. Friesner, R.A.; Murphy, R.B.; Repasky, M.P.; Frye, L.L.; Greenwood, J.R.; Halgren, T.A.; Sanschagrin, P.C.; Mainz, D.T. Extra precision glide: Docking and scoring incorporating a model of hydrophobic enclosure for protein-ligand complexes. J. Med. Chem. 2006, 49, 6177-6196. [CrossRef] [PubMed]

24. Friesner, R.A.; Murphy, R.B.; Repasky, M.P.; Sherman, B.W. Use of the Glide extra precision methodology for docking and scoring. Abstr. Pap. Am. Chem. S. 2006, 232, 42.

25. Bitencourt-Ferreira, G.; de Azevedo, W.F. Docking with SwissDock. In Docking Screens for Drug Discovery; de Azevedo, W.F., Jr., Ed.; Springer: New York, NY, USA, 2019; pp. 189-202.

26. Fischer, E. Einfluss der Configuration auf die Wirkung der Enzyme. Ber. Dtsch. Chem. Ges. 1894, 27, $2985-2993$. [CrossRef]

27. Sherman, W.; Day, T.; Jacobson, M.P.; Friesner, R.A.; Farid, R. Novel procedure for modeling ligand/receptor induced fit effects. J. Med. Chem. 2006, 49, 534-553. [CrossRef]

28. Morris, G.M.; Huey, R.; Lindstrom, W.; Sanner, M.F.; Belew, R.K.; Goodsell, D.S.; Olson, A.J. AutoDock4 and AutoDockTools4: Automated Docking with Selective Receptor Flexibility. J. Comput. Chem. 2009, 30, 2785-2791. [CrossRef]

29. Nabuurs, S.B.; Wagener, M.; De Vlieg, J. A flexible approach to induced fit docking. J. Med. Chem. 2007, 50, 6507-6518. [CrossRef]

30. Amaro, R.E.; Baudry, J.; Chodera, J.; Demir, Ö.; McCammon, J.A.; Miao, Y.; Smith, J.C. Ensemble Docking in Drug Discovery. Biophys. J. 2018, 114, 2271-2278. [CrossRef]

31. Sekharan, S.; Ertem, M.Z.; Zhuang, H.Y.; Block, E.; Matsunami, H.; Zhang, R.N.; Wei, J.N.; Pan, Y.; Batista, V.S. QM/MM Model of the Mouse Olfactory Receptor MOR244-3 Validated by Site-Directed Mutagenesis Experiments. Biophys. J. 2014, 107, 5-8. [CrossRef]

32. Ahmed, L.; Zhang, Y.T.; Block, E.; Buehl, M.; Corr, M.J.; Cormanich, R.A.; Gundala, S.; Matsunami, H.; O'Hagan, D.; Ozbil, M.; et al. Molecular mechanism of activation of human musk receptors OR5AN1 and OR1A1 by (R)-muscone and diverse other musk-smelling compounds. Proc. Natl. Acad. Sci. USA 2018, 115, 3950-3958. [CrossRef]

33. Zanatta, G.; Nunes, G.; Bezerra, E.M.; da Costa, R.F.; Martins, A.; Caetano, E.W.S.; Freire, V.N.; Gottfried, C. Antipsychotic Haloperidol Binding to the Human Dopamine D3 Receptor: Beyond Docking Through QM/MM Refinement Toward the Design of Improved Schizophrenia Medicines. Acs Chem. Neurosci. 2014, 5, 1041-1054. [CrossRef]

34. Chien, E.Y.T.; Liu, W.; Zhao, Q.A.; Katritch, V.; Han, G.W.; Hanson, M.A.; Shi, L.; Newman, A.H.; Javitch, J.A.; Cherezov, V.; et al. Structure of the Human Dopamine D3 Receptor in Complex with a D2/D3 Selective Antagonist. Science 2010, 330, 1091-1095. [CrossRef] [PubMed]

35. Newcomer, J.W. Second-generation (atypical) antipsychotics and metabolic effects - A comprehensive literature review. Cns Drugs 2005, 19, 1-93. [CrossRef] [PubMed]

36. Cho, A.E.; Guallar, V.; Berne, B.J.; Friesner, R. Importance of accurate charges in molecular docking: Quantum mechanical/molecular mechanical (QM/MM) approach. J. Comput. Chem. 2005, 26, 915-931. [CrossRef] [PubMed]

37. Cho, A.E.; Rinaldo, D. Extension of QM/MM Docking and its Applications to Metalloproteins. J. Comput. Chem. 2009, 30, 2609-2616. [CrossRef] [PubMed]

38. Chung, J.Y.; Hah, J.M.; Cho, A.E. Correlation between Performance of QM/MM Docking and Simple Classification of Binding Sites. J. Chem. Inf. Model 2009, 49, 2382-2387. [CrossRef]

39. Burger, S.K.; Thompson, D.C.; Ayers, P.W. Quantum Mechanics/Molecular Mechanics Strategies for Docking Pose Refinement: Distinguishing between Binders and Decoys in Cytochrome c Peroxidase. J. Chem. Inf. Model 2011, 51, 93-101. [CrossRef]

40. Chaskar, P.; Zoete, V.; Rohrig, U.F. On-the-Fly QM/MM Docking with Attracting Cavities. J. Chem. Inf. Model 2017, 57, 73-84. [CrossRef]

41. Beuming, T.; Sherman, W. Current Assessment of Docking into GPCR Crystal Structures and Homology Models: Successes, Challenges, and Guidelines. J. Chem. Inf. Model. 2012, 52, 3263-3277. [CrossRef] 
42. Yu, J.H.; Mannes, P.; Jung, Y.H.; Ciancetta, A.; Bitant, A.; Lieberman, D.I.; Khaznadar, S.; Auchampach, J.A.; Gao, Z.G.; Jacobson, K.A. Structure activity relationship of 2-arylalkynyl-adenine derivatives as human A(3) adenosine receptor antagonists. Medchemcomm 2018, 9, 1920-1932. [CrossRef]

43. Kim, M.; Cho, A.E. Incorporating QM and solvation into docking for applications to GPCR targets. Phys. Chem. Chem. Phys. 2016, 18, 28281-28289. [CrossRef]

44. Gascon, J.A.; Batista, V.S. QM/MM study of energy storage and molecular rearrangements due to the primary event in vision. Biophys. J. 2004, 87, 2931-2941. [CrossRef] [PubMed]

45. Send, R.; Sundholm, D. Stairway to the Conical Intersection: A Computational Study of the Retinal Isomerization. J. Phys. Chem. A 2007, 111, 8766-8773. [CrossRef] [PubMed]

46. Send, R.; Sundholm, D. Coupled-cluster studies of the lowest excited states of the 11-cis-retinal chromophore. Phys. Chem. Chem. Phys. 2007, 9, 2862-2867. [CrossRef] [PubMed]

47. Schick, G.A.; Cooper, T.M.; Holloway, R.A.; Murray, L.P.; Birge, R.R. Energy storage in the primary photochemical events of rhodopsin and isorhodopsin. Biochemistry 1987, 26, 2556-2562. [CrossRef]

48. Cooper, A. Energetics of rhodopsin and isorhodopsin. FEBS Lett. 1979, 100, 382-384. [CrossRef]

49. Cooper, A. Energy uptake in the first step of visual excitation. Nature 1979, 282, 531-533. [CrossRef]

50. Altun, A.; Yokoyama, S.; Morokuma, K. Mechanism of Spectral Tuning Going from Retinal in Vacuo to Bovine Rhodopsin and its Mutants: Multireference ab Initio Quantum Mechanics/Molecular Mechanics Studies. J. Phys. Chem. B 2008, 112, 16883-16890. [CrossRef]

51. Altun, A.; Yokoyama, S.; Morokuma, K. Spectral tuning in visual pigments: An ONIOM(QM: MM) study on bovine rhodopsin and its mutants. J. Phys. Chem. B 2008, 112, 6814-6827. [CrossRef]

52. Sekharan, S.; Altun, A.; Morokuma, K. Photochemistry of Visual Pigment in a G(q) Protein-Coupled Receptor (GPCR)-Insights from Structural and Spectral Tuning Studies on Squid Rhodopsin. Chem. Eur. J. 2010, 16, 1744-1749. [CrossRef]

53. Sekharan, S.; Morokuma, K. Drawing the Retinal Out of Its Comfort Zone: An ONIOM(QM/MM) Study of Mutant Squid Rhodopsin. J. Phys. Chem. Lett. 2010, 1, 668-672. [CrossRef]

54. Hernandez-Rodriguez, E.W.; Sanchez-Garcia, E.; Crespo-Otero, R.; Montero-Alejo, A.L.; Montero, L.A.; Thiel, W. Understanding Rhodopsin Mutations Linked to the Retinitis pigmentosa Disease: A QM/MM and DFT/MRCI Study. J. Phys. Chem. B 2012, 116, 1060-1076. [CrossRef] [PubMed]

55. Rakoczy, E.P.; Kiel, C.; McKeone, R.; Stricher, F.; Serrano, L. Analysis of Disease-Linked Rhodopsin Mutations Based on Structure, Function, and Protein Stability Calculations. J. Mol. Biol. 2011, 405, 584-606. [CrossRef] [PubMed]

56. Stojanovic, A.; Hwa, J. Rhodopsin and retinitis pigmentosa: Shedding light on structure and function. Receptors Channels 2002, 8, 33-50. [CrossRef] [PubMed]

57. Chung, W.C.; Nanbu, S.; Ishida, T. QM/MM Trajectory Surface Hopping Approach to Photoisomerization of Rhodopsin and Isorhodopsin: The Origin of Faster and More Efficient Isomerization for Rhodopsin. J. Phys. Chem. B 2012, 116, 8009-8023. [CrossRef]

58. Mattle, D.; Kuhn, B.; Aebi, J.; Bedoucha, M.; Kekilli, D.; Grozinger, N.; Alker, A.; Rudolph, M.G.; Schmid, G.; Schertler, G.F.X.; et al. Ligand Channel in Pharmacologically stabilized Rhodopsin. Proc. Natl. Acad. Sci. USA 2018, 115, 3640-3645. [CrossRef]

59. Pedraza-Gonzalez, L.; De Vico, L.; Marin, M.D.; Fanelli, F.; Olivucci, M. a-ARM: Automatic Rhodopsin Modeling with Chromophore Cavity Generation, Ionization State Selection, and External Counterion Placement. J. Chem. Theory Comput. 2019, 15, 3134-3152. [CrossRef]

60. Fedorov, D.G.; Kitaura, K. Extending the power of quantum chemistry to large systems with the fragment molecular orbital method. J. Phys. Chem. A 2007, 111, 6904-6914. [CrossRef]

61. Kitaura, K.; Ikeo, E.; Asada, T.; Nakano, T.; Uebayasi, M. Fragment molecular orbital method: An approximate computational method for large molecules. Chem. Phys. Lett. 1999, 313, 701-706. [CrossRef]

62. Fedorov, D.G.; Nagata, T.; Kitaura, K. Exploring chemistry with the fragment molecular orbital method. Phys. Chem. Chem. Phys. 2012, 14, 7562-7577. [CrossRef]

63. Hayashi, S.; Taikhorshid, E.; Schulten, K. Photochemical Reaction Dynamics of the Primary Event of Vision Studied by Means of a Hybrid Molecular Simulation. Biophys. J. 2009, 96, 403-416. [CrossRef]

64. Heifetz, A.; Aldeghi, M.; Chudyk, E.I.; Fedorov, D.G.; Bodkin, M.J.; Biggin, P.C. Using the fragment molecular orbital method to investigate agonist-orexin-2 receptor interactions. Biochem. Soc. T. 2016, 44, 574-581. [CrossRef] [PubMed] 
65. Heifetz, A.; Chudyk, E.I.; Gleave, L.; Aldeghi, M.; Cherezov, V.; Fedorov, D.G.; Biggin, P.C.; Bodkin, M.J. The Fragment Molecular Orbital Method Reveals New Insight into the Chemical Nature of GPCR-Ligand Interactions. J. Chem. Inf. Model 2016, 56, 159-172. [CrossRef] [PubMed]

66. Heifetz, A.; James, T.; Southey, M.; Morao, I.; Aldeghi, M.; Sarrat, L.; Fedorov, D.G.; Bodkin, M.J.; Townsend-Nicholson, A. Characterising GPCR-ligand interactions using a fragment molecular orbital-based approach. Curr. Opin. Struc. Biol. 2019, 55, 85-92. [CrossRef] [PubMed]

67. Heifetz, A.; Trani, G.; Aldeghi, M.; MacKinnon, C.H.; McEwan, P.A.; Brookfield, F.A.; Chudyk, E.I.; Bodkin, M.; Pei, Z.H.; Burch, J.D.; et al. Fragment Molecular Orbital Method Applied to Lead Optimization of Novel Interleukin-2 Inducible T-Cell Kinase (ITK) Inhibitors. J. Med. Chem. 2016, 59, 4352-4363. [CrossRef]

68. Morao, I.; Fedorov, D.G.; Robinson, R.; Southey, M.; Townsend-Nicholson, A.; Bodkin, M.J.; Heifetz, A. Rapid and Accurate Assessment of GPCR-Ligand Interactions Using the Fragment Molecular Orbital-Based Density-Functional Tight-Binding Method. J. Comput. Chem. 2017, 38, 1987-1990. [CrossRef]

69. Tokiwa, T.; Nakano, S.; Yamamoto, Y.; Ishikawa, T.; Ito, S.; Sladek, V.; Fukuzawa, K.; Mochizuki, Y.; Tokiwa, H.; Misaizu, F.; et al. Development of an Analysis Toolkit, AnalysisFMO, to Visualize Interaction Energies Generated by Fragment Molecular Orbital Calculations. J. Chem. Inf. Model 2019, 59, 25-30. [CrossRef]

70. Watanabe, C.; Watanabe, H.; Okiyama, Y.; Takaya, D.; Fukuzawa, K.; Tanaka, S.; Honma, T. Development of an automated fragment molecular orbital (FMO) calculation protocol toward construction of quantum mechanical calculation database for large biomolecules. Chem-Bio Inform. J. 2019, 19, 5-18. [CrossRef]

(C) 2020 by the authors. Licensee MDPI, Basel, Switzerland. This article is an open access article distributed under the terms and conditions of the Creative Commons Attribution (CC BY) license (http://creativecommons.org/licenses/by/4.0/). 